hep-th/9605174, PUPT-1600

\title{
Some Aspects of Massive World-Brane Dynamics
}

\author{
Vijay Balasubramanian and Igor R. Klebanov* \\ Joseph Henry Laboratories, \\ Princeton University, Princeton, NJ 08544
}

September 7, 2018

\begin{abstract}
We study the internal dynamics of Ramond-Ramond solitons excited far from the BPS limit by leading Regge trajectory open strings. The simplest world volume process for such strings is splitting into two smaller pieces, and we calculate the corresponding decay rates. Compared to the conventional open superstring, the splitting of states polarized parallel to the brane is suppressed by powers of logarithms of the energy. The rate for states polarized transverse to the brane decreases with increasing energy. We also calculate the static force between a D-brane excited by a massive open string and an unexcited D-brane parallel to it. The result shows that transversely polarized massive open strings endow D-branes with a size of order the string scale.
\end{abstract}

\section{Introduction}

Open strings with Dirichlet boundary conditions provide a conformal field theory description of Ramond-Ramond solitons [罒]. In this paper we study the dynamics of these objects moved far from the BPS limit by states on the leading Regge trajectory. We are motivated in this study by recent progress in describing black hole thermodynamics using D-branes [2] - [10]. In order to have a thermodynamic description, excitations of an object must be able to equipartition their energy at a sufficiently rapid rate. The D-brane black holes constructed thus far involve multiple intersecting branes and are difficult to handle in such a dynamical context. On the other hand, individual p-branes have classical horizons [11] and in some cases have entropies that scale in the same way as the Bekenstein-Hawking formula [12, 13]. Therefore, we hope to gain some insight into generic issues like equipartition of energy by studying individual RR solitons.

*vijayb,klebanov@puhep1.princeton.edu 
To this end, we excite D-branes far from the BPS limit by attaching massive open strings on the leading Regge trajectory to them. The importance of massive excitations on the world volume is exhibited by scattering of closed strings off Dbranes [14, 15, 16]. There, the massive intermediate states give rise to an infinite sequence of $s$-channel poles. It is precisely these excitations that endow D-branes with an effective transverse size which increases with the energy of the probe and is of order $\sqrt{\alpha^{\prime}}$. The dynamics of the massive modes is not constrained by supersymmetry, and in this paper we explore some of its features at weak coupling. We find it important to distinguish between states that are polarized in the longitudinal and in the transverse directions. The former classically correspond to strings that lie entirely within the brane, while the latter describe strings "hanging" off the brane. We will find that there are major differences in the behavior of these two kinds of states. Not surprisingly, it is only the transversely polarized states that are responsible for the energy-dependent thickness of D-branes (we demonstrate this is Section 4).

The dominant world volume process for the massive strings is splitting into pairs of lighter states. The rate for the splitting process can be studied by extracting the imaginary part of the one-loop diagram for strings attached to a D-brane. We find that the decay rates are suppressed compared to the results for a conventional superstring. States polarized parallel to the brane have decay rates suppressed by powers of logarithms of the energy. States polarized transverse to the brane have rates that often decrease with increasing energy. Furthermore, strings attached to a 0-brane simply cannot decay via splitting into two smaller pieces - the leading channels are higher order in the string coupling. The source of these unusual effects is the fact that all open string momenta must lie within the world volume.

In Section 2 we calculate the one-loop amplitude for a D-brane. We employ it in Section 3 to extract the decay rates of highly excited string states. In Section 1 the one-loop amplitude is used to study forces between parallel D-branes. Evidence is displayed that excitations transverse to a brane give it a finite extent which increases with the excitation level and is of order the string length. Implications of the results are discussed in Section 5 .

\section{D-Brane Loop Amplitude}

The decay rates we are interested in are most easily calculated as the imaginary part of the annulus diagram in Figure 1 . As an added benefit, when the boundaries of the annulus are attached to different parallel branes, the diagram gives the interaction potential between them. We will calculate the annulus amplitude for NS states on the leading Regge trajectory by modifying the results of [2], obtained for the conventional open superstring.

It is useful to review the analysis of the one-loop two-point amplitude of the open superstring. On the annulus we can choose vertex operators of ghost number zero [19].

\footnotetext{
${ }^{1}$ Our strings are oriented, so the Möbius strip is absent. The nonplanar contribution is expected to be highly suppressed, and contains closed string intermediate states [17, 18, 19].
} 


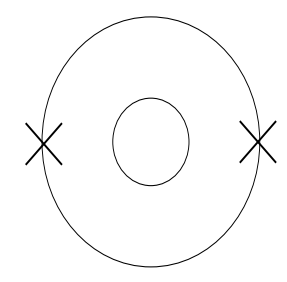

Figure 1: When both boundaries are placed on the same brane, the imaginary part of this diagram gives the decay rate of a state via splitting into pairs of lighter states attached to the brane. When the boundaries are on different branes the diagram computes the static interaction potential between an excited brane and another in its ground state.

For leading Regge trajectory states with $m^{2}=2 l$ and $J=l+1$ for $l \geq 0$ this gives [20]:

$$
\begin{array}{r}
V_{(2 l, l+1)}=\frac{\xi_{\mu \nu_{1} \cdots \nu_{l}}}{\sqrt{l !}\left[\partial X^{\mu} \partial X^{\nu_{1}} \cdots \partial X^{\nu_{l}}+\sum_{j=1}^{l} \partial \psi^{\nu_{j}} \psi^{\mu} \partial X^{\nu_{1}} \cdots \partial X^{\nu_{j-1}} \partial X^{\nu_{j+1}} \cdots \partial X^{\nu_{l}}\right.} \\
\left.+i(k \cdot \psi) \psi^{\mu} \partial X^{\nu_{1}} \cdots \partial X^{\nu_{l}}\right] e^{i k \cdot X}
\end{array}
$$

Here $\partial$ stands for $\partial_{\tau}$, the derivative with respect to the world-sheet time and $\xi$ is a totally symmetric polarization tensor that is transverse to the momentum $k$ and traceless.

The two-point annulus amplitude for these vertex operators has the general structure:

$$
A=\sum_{s} \eta_{s} \int d \omega F_{s}(\omega)\left\langle V_{1} V_{2}\right\rangle_{s}
$$

where $\sum_{s}$ is the sum over spin structures, $\eta_{s}$ is relative phase between spin structures and $d \omega$ is a measure on the annulus. The factors $F_{s}$ include the trace over the momentum running around the loop and the partition function of oscillators, and the correlation functions are evaluated separately in each spin structure. It is convenient to work in variables $w=\rho_{1} \rho_{2}$ and $\nu=\ln \rho_{1} / \ln w$ where $\rho_{1}$ and $\rho_{2}$ are the standard variables on the strip introduced in Chapter 8 of [21]. Then $\ln w$ is the world-sheet time elapsed in travelling around the loop. Evaluating the correlation functions and doing the sum over spin structures gives [20]:

$$
\begin{aligned}
A & =\mathcal{N} \int_{0}^{1} d \nu \int_{\epsilon}^{\infty} d(-\ln w)\left(\frac{1}{-\ln w}\right)^{4} \Psi(\nu, w)^{2 l} \Omega(\nu, w)^{l-1} \\
\mathcal{N} & =2 \pi^{2}(2 \pi)^{3} \xi^{2} l
\end{aligned}
$$

where $\Psi=\langle X X\rangle$ and $\Omega=\langle\partial X \partial X\rangle$ are the bosonic correlators; $\xi^{2}$ stands for $\xi_{\mu \nu_{1} \cdots \nu_{l}}^{(1)} \xi_{\mu \nu_{1} \cdots \nu_{l}}^{(2)}$. As usual, we have introduced $\epsilon$, a cutoff that is necessary to define the integral over the world-sheet momenta and is taken to zero at the end of the calculation. The integration over these momenta gives rise to a factor $(-2 \pi / \ln w)^{D / 2}=$ 
$(-2 \pi / \ln w)^{5}$ in Eq. 3. 2 Note that the physical meaning of the annulus amplitude is the one-loop mass shift. The fact that this vanishes for the gauge boson ( $l=0$ state) is in accord with gauge invariance.

In order to generalize to D-brane amplitudes, note that the partition functions and correlators appearing in Eq. 2 factorize into products of fermionic and bosonic parts. We will first study the effect of Dirichlet boundary conditions on bosons and then on fermions. Dirichlet boundary conditions are implemented on some coordinates by flipping the sign of the corresponding left-moving bosons. This yields the following mode expansion describing strings stretched between parallel k-branes.

$$
\begin{aligned}
0 \leq \mu \leq k: \quad X^{\mu} & =X_{0}^{\mu}+p^{\mu} \tau+i \sum_{n \neq 0} \frac{1}{n} \alpha_{n}^{\mu} \cos (n \sigma) e^{-i n \tau} \\
k+1 & \leq \mu \leq D: \quad X^{\mu}=X_{0}^{\mu}+\frac{Y^{\mu}}{\pi} \sigma+\sum_{n \neq 0} \frac{1}{n} \alpha_{n}^{\mu} \sin (n \sigma) e^{-i n \tau}
\end{aligned}
$$

Since the branes are stationary, we do not integrate over $Y$, which measures the offset between them. Furthermore, neither the vertex operators on the boundary of Figure 1 nor the states running around the loop can carry momentum in the Dirichlet directions. Finally, the change in sign of the left moving boson implies that the operators $\partial X^{\mu}$ appearing in Equation 11 should be read as $\partial_{\tau} X^{\mu}$ (tangential derivatives) for Neumann coordinates and $\partial_{\sigma} X^{\mu}$ (normal derivatives) for Dirichlet coordinates.

Since the Dirichlet boundary conditions do not affect the spectrum of the open strings, the bosonic contribution to the partition functions in Eq. 2 is unchanged. However, the restriction of world-sheet momenta to lie parallel to the brane has important effects. First of all, the trace over world-sheet momenta produces a factor of $(-2 \pi / \ln w)^{(k+1) / 2)}$ instead of $(-2 \pi / \ln w)^{D / 2}$. Furthermore, the separation $Y^{\mu}$ between the boundaries contributes a factor of $\exp \left(Y^{2} \ln w / 2 \pi^{2}\right)$. p Finally, the bosonic correlators are modified as discussed in [23, 24]. Further subtleties arise when the separation $Y^{\mu}$ is not orthogonal to the polarization tensor. We will avoid them by only considering states polarized transverse to both the momentum $k$ and the separation $Y$. In any case, we are mostly interested in the case $Y=0$ for which the additional transversality requirement does not pose a constraint.

On the other hand, the fermionic contributions to the amplitude are completely unchanged by the imposition of Dirichlet boundary condition. To see this, remember that Dirichlet boundary conditions are implemented by changing the sign of the left moving bosons, and so supersymmetry instructs us to flip the sign of the left-moving fermions also. Now, the NS and R sectors of the amplitude arise from the relative sign of the left and right moving fermions at the ends of the string. But changing the sign of the left-mover only changes the conventional overall sign between left-moving and right-moving fermions. Therefore imposing Dirichlet boundary conditions on some coordinates has no effect on the fermionic partition function and correlators.

\footnotetext{
${ }^{2}$ The additional factor of $(-\ln w)$ in Eq. 3 arises from the measure for $d \nu$ [21].

${ }^{3}$ The separation between the branes contributes $Y^{2} /\left(4 \pi^{2} \alpha^{\prime}\right)$ to $L_{0}$ [22]. Setting $\alpha^{\prime}=1 / 2$, this adds a contribution $w^{Y^{2} /\left(2 \pi^{2}\right)}$ to the loop amplitude.
} 
Using the factorization of the bosonic and fermionic contributions to the one-loop amplitude and the observations made above, the results of [20] are readily combined with the correlators derived in [23] to yield D-brane amplitudes. It is easiest to work with states polarized entirely parallel or perpendicular to a brane. (As discussed above, the states are also taken to be orthogonal to the separation $Y^{\mu}$.) The one-loop amplitudes for leading Regge trajectory states at level $l$ on a k-brane are:

$$
\begin{aligned}
A_{(N, D)} & =\mathcal{N}_{(N, D)} \int_{0}^{1} d \nu \int_{\epsilon}^{\infty} d(-\ln w)\left(\frac{1}{-\ln w}\right)^{\frac{k-1}{2}} e^{\left(Y^{2} / 2 \pi^{2}\right) \ln w} \Psi^{2 l} \Omega_{(N, D)}^{l-1} \\
\Psi & =(-\ln w) \frac{\theta_{1}(\nu \mid \tau)}{\theta_{1}^{\prime}(0 \mid \tau)}=\frac{e^{(1 / 2) \nu(\nu-1) \ln w}}{f(w)^{3}} \sum_{n=-\infty}^{\infty}(-1)^{n} \rho_{1}^{n(n+1) / 2} \rho_{2}^{n(n-1) / 2} \\
\Omega_{N} & =\frac{-1}{(\ln w)^{2}} \partial_{\nu}^{2} \ln \theta_{1}(\nu \mid \tau)=\frac{-1}{\ln w}+\sum_{n=1}^{\infty} n\left[\frac{\rho_{1}^{n}+\rho_{2}^{n}}{1-w^{n}}\right] \\
\Omega_{D} & =\Omega_{N}+\frac{1}{\ln w}=\sum_{n=1}^{\infty} n\left[\frac{\rho_{1}^{n}+\rho_{2}^{n}}{1-w^{n}}\right] \\
\mathcal{N}_{(N, D)} & =\left(2 \pi^{2}\right)(2 \pi)^{3}(2 \pi)^{(k+1-D) / 2} l \xi_{(N, D)}^{2} \quad ; \quad f(w)=\prod_{i=1}^{\infty}\left(1-w^{i}\right)
\end{aligned}
$$

Here $\rho_{1}=\exp (\nu \ln w)$ and $\rho_{2}=\exp ((1-\nu) \ln w)$ with $w=\rho_{1} \rho_{2}$ and $\tau=-2 \pi i / \ln w$. We have used standard series expansions for the $\theta$ functions arising in the $\Psi$ and $\Omega$ correlators. The subscripts $\mathrm{N}$ and $\mathrm{D}$ refer to states that are polarized entirely in the longitudinal (Neumann) or transverse (Dirichlet) directions respectively. The difference of a $-1 / \ln w$ between $\Omega_{N}$ and $\Omega_{D}$ will have a profound effect on the relative decay rates.

\section{$3 \quad$ Splitting Rates}

The splitting rates of highly excited string states can be extracted from Eq. 5. In the old operator formalism, the overall amplitude is written as $\operatorname{Tr}\left(\rho_{1}^{L_{0}} V_{1} \rho_{2}^{L_{0}} V_{2}\right)$. So, the term proportional to $\rho_{1}^{p} \rho_{2}^{q}$ in a power series expansion of the integrand contains the intermediate channel of levels $p$ and $q$. f Performing such an expansion to isolate the channels gives:

$$
\begin{aligned}
A_{N} & =\mathcal{N}_{N} \sum_{p, q \geq 0} \sum_{r=0}^{l-1} C_{p q}^{r} \int_{0}^{1} d \nu \int_{\epsilon}^{\infty} d(-\ln w)\left(\frac{1}{-\ln w}\right)^{r+(k-1) / 2} e^{B(\nu, p, q) \ln (\Psi 0)} \\
A_{D} & =\mathcal{N}_{D} \sum_{p, q \geq 0} D_{p q} \int_{0}^{1} d \nu \int_{\epsilon}^{\infty} d(-\ln w)\left(\frac{1}{-\ln w}\right)^{(k-1) / 2} e^{B(\nu, p, q) \ln w} \\
B(\nu, p, q) & =l(\nu-1) \nu+p \nu+q(1-\nu)+\frac{Y^{2}}{2 \pi^{2}}
\end{aligned}
$$

Here the index $r$ is associated with powers of $\ln (w)$ that arise from expansion of $\Omega_{N}^{l-1}$ in the case of parallel polarizations. The coefficients $C_{p q}^{r}$ and $D_{p q}$ are determined

\footnotetext{
${ }^{4}$ See [17] and [25] for similar analyses of conventional open strings.
} 


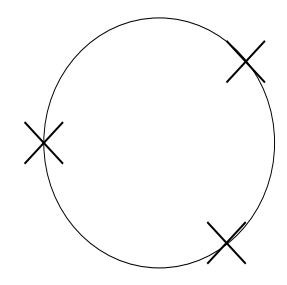

Figure 2: The leading channel for splitting of strings attached to a brane is this three point diagram.

by expanding the integrand of Eq. 5 in powers of $\rho_{1}$ and $\rho_{2}$. When $B<0$ this (Euclidean) amplitude diverges, and it is easily seen that this can only happen when $\sqrt{l}>\sqrt{p+Y^{2} / 4 \pi^{2}}+\sqrt{q+Y^{2} / 4 \pi^{2}}$ as should be expected for a threshold cut. . Below, we set $Y^{2}=0$ and study the imaginary part of the amplitude arising from these threshold cuts to derive the decay rate of highly excited string states on D-branes.

\subsection{Transverse Polarizations}

For decays of traversely polarized states the key point is that the absence of a leading $(-1 / \ln w)$ in $\Omega_{D}$ implies that $p+q \geq l-1$ in $A_{D}$. The threshold condition for the $(p, q)$ channel requires $\sqrt{l}>\sqrt{p}+\sqrt{q}$ so that $l>p+q+2 \sqrt{p q}$. The only simultaneous solutions of these equations are $(p, q)=\{(l-1,0),(0, l-1)\}$. Thus, the only decay channel for a transversely polarized leading Regge trajectory state at level $l$ is into a leading Regge trajectory state at level $l-1$ and a massless state.

This can also be seen from the disk diagram in Figure 2 representing a given decay channel. Suppose one of the boundary operators is a level $l$, transversely polarised, leading Regge trajectory state and the other two are states at levels $p$ and $q$. Then, by conservation of energy, $\sqrt{l} \geq \sqrt{p}+\sqrt{q}$. Next, in the center of mass frame, the first vertex operator carries an "angular momentum" $l+1$ f which must be conserved by the decay products. The angular momentum carried by a stationary transversely polarized state cannot be made up from the relative motions of the decay products on the brane. So, because the intrinsic angular momentum carried by a state at level $p$ is at most $p+1$, we must have $(p+1)+(q+1) \geq l+1$ giving $p+q \geq l-1$. This reproduces the selection rules for decays of transversely polarized states derived in the previous paragraph. 

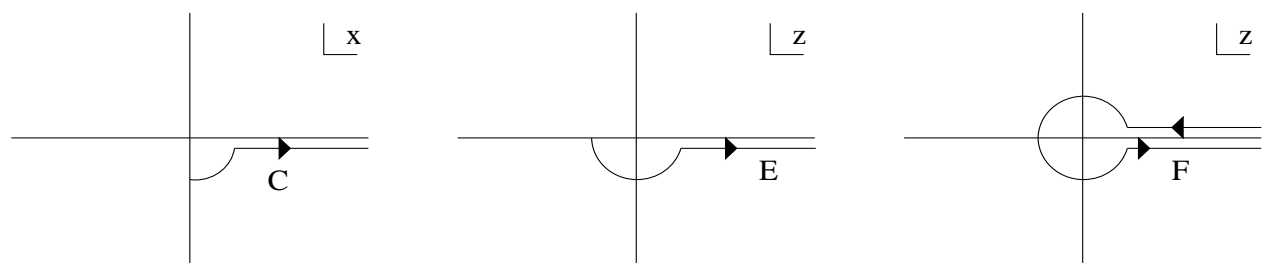

Figure 3: Integration contours for decay amplitudes.

\section{$3.2 k>0$}

For a k-brane with $k>0$ the decay amplitude is most readily extracted by continuing Eq. 11 back to a Minkowski world-sheet and examining the imaginary part. Setting $-\ln w=i x$, the integral in the $(p, q)$ channel is:

$$
I_{p q}=\int d \nu \int_{C} d x i(i x)^{(1-k) / 2} e^{-i x B(\nu, p, q)}
$$

where $\mathrm{C}$ is the contour in Figure 3.1] The integral can be carried out by rotating the contour to the positive or negative imaginary axes. When $B>0$, the contour must be rotated down, giving a purely real integral. When $B<0$, the contour must be rotated up and picks up a contribution from the pole at the origin. Letting $\nu_{+}$and $\nu_{-}$be the two zeroes of $B(\nu, p, q)$, and performing some rescalings, the imaginary part of the amplitude in the $(p, q)$ channel is:

$$
\begin{aligned}
\operatorname{Im}\left(A_{p q}\right) & =\mathcal{N}_{D} D_{p q} \int_{\nu_{-}}^{\nu_{+}} d \nu|B(\nu, p, q)|^{(k-3) / 2} \operatorname{Im}(J) \\
\operatorname{Im}(J) & =-\operatorname{Im}\left[\int_{E} d z(-z)^{(1-k) / 2} e^{-z}\right] \\
& =\frac{-1}{2} \operatorname{Im}\left[\int_{F} d z(-z)^{(1-k) / 2} e^{-z}\right]=\frac{\pi}{\Gamma((k-1) / 2)}
\end{aligned}
$$

The contours E and F are shown in Figure 3 and the last equality in Eq. 15 follows from Hänkel's formula for the Gamma function [26]. Defining $a=\left(\nu_{+}-\nu_{-}\right) / 2$, the

\footnotetext{
${ }^{5}$ In fact, because we are working with a Euclidean world-sheet, the amplitudes are formally real. However, we will shortly continue back to a Minkowski world-sheet in order to extract the decay amplitudes.

${ }^{6}$ More precisely, this means that the bosonic part of this vertex operator contains $l+1 \partial_{n} X$ factors.

${ }^{7}$ The integral starts on the negative imaginary axis in order to make the trace over world-sheet Minkowski momenta well defined. The offset is taken to zero at the end of the calculation. See [17] for a similar treatment of the bosonic string.

${ }^{8}$ For $k=1$ there is apparently no divergence in Eq. 11 at $\ln w \approx 0$. However, the case of a 1-brane is rather delicate since because the imaginary part of the amplitude for $k=1+\epsilon$ is non-zero while it vanishes for $k=1-\epsilon$. The physical prescription is to define the decay amplitude for a 1-brane by a continuation from $k=1+\epsilon$ as will be seen below from the agreement of the computed decay rate with phase space arguments.
} 
remaining integral can be done as in [17] to find the decay rate into the $(p, q)$ channel:

$$
\operatorname{Im}\left(A_{p q}\right)=\mathcal{N}_{D} D_{p q} \frac{\pi^{3 / 2} l^{(k-3) / 2} a^{k-2}}{\Gamma(k / 2)}
$$

Finally, $a=1 /(2 l)$ for the channels $(l-1,0)$ and $(0, l-1)$. Combining this with $D_{l-1,0}=1$ in Eq. 11] and the relativistic normalization $\xi_{D}^{2}=1 / 2 \sqrt{l}$ [17], yields the decay rate:

$$
\operatorname{Im}\left(A_{D}\right)=\left(\frac{1}{2 \pi}\right)^{4} \frac{8 \pi^{5} \pi^{3 / 2}}{\Gamma(k / 2)} \sqrt{l}\left(\frac{\pi}{2 l}\right)^{(k-1) / 2}
$$

Surprisingly, for $k>2$ the decay rate actually decreases as the level $l$ increases, while for $k=2$ the rate is independent of $l$. Naively one might have expected that the decay rate grows with the excitation number. This does not happen due to the extremely constrained phase space for the decays of transversely polarized states. Indeed, in the center of mass frame, energy conservation for the decay of a level $l$ state into states of levels $l-1$ and 0 requires the decay products to carry spatial momentum with magnitude of order $1 / \sqrt{l}$. The available phase space for states with this momentum scales as $(1 / l)^{(k-1) / 2}$ and explains the final suppression factor in Eq. 17. In particular, note that on a 1-brane energy conservation gives a decay phase space consisting of precisely two points. The volume of phase space is therefore independent of energy. This agrees with the lack of suppression in Eq. 17 for $k=1$.

\subsection{1 $k=0$}

When $k=0$, the absence of a divergence in Eq. 11 at $\ln (w) \approx 0$ tells us that the procedure of the previous section will not give us an imaginary part. So the rate for splitting of a level $l$ state on a 0-brane into two smaller pieces is exactly zero. Indeed, for a 0-brane, conservation of energy requires that $\sqrt{l}=\sqrt{p}+\sqrt{q}$, which is in immediate conflict with the selection rule $\sqrt{l}>\sqrt{p}+\sqrt{q}$. Thus, there are no allowed decay channels. This statement also applies to subleading Regge trajectories since it follows from energy conservation on a $0+1$ dimensional worldvolume. Therefore, excited states of 0 -branes are exceptionally stable - they decay via higher order diagrams involving more powers of the string coupling. The physical implication of this fact is the relative stability of excitations of ten-dimensional RRcharged black holes - they can only decay via emission of closed string states.

\subsection{Parallel Polarizations}

Because the expansion of $\Omega_{N}^{l-1}$ in powers of $\rho_{1}^{p}$ and $\rho_{2}^{q}$ contains all terms with $p+q \leq$ $l-1$, all channels that are permitted by the threshold condition contribute to the decays. The combinatorics of the channel-by-channel sum is very hard and so we instead carry out an asymptotic analysis of the total decay rate [18.

The key point is that the imaginary part of the one-loop amplitude arises from the nature of the divergence near $w=1$. The analysis is easiest in annular variables $\nu$ and 
$\ln q=2 \pi^{2} / \ln w$. Transforming to these variables, and using the modular properties of $\theta$ functions yields the amplitude:

$$
A_{N}=\frac{\mathcal{N}_{N}}{2 \pi^{2}} \int_{0}^{1} d \nu \int_{0}^{1} \frac{d q}{q}\left(\frac{-\ln q}{2 \pi^{2}}\right)^{(k+1-D) / 2} e^{Y^{2} / \ln (q)}\left(\frac{\theta_{1}(\nu \mid \tau)}{\theta_{1}^{\prime}(0 \mid \tau)}\right)^{2 l}\left(-\partial_{\nu}^{2} \ln \theta_{1}(\nu \mid \tau)\right)^{l-1}
$$

We now set $Y^{2}=0$ because we are interested in decays of states attached to a single brane. The behaviour of the amplitude near $q=0$ (the ultraviolet region of the open string channel) determines the decay rate. Expanding the amplitude near $q=0$ using standard $\theta$ function identities gives:

$$
A_{N}=\frac{\mathcal{N}_{N}}{2 \pi^{2}} \int_{0}^{1} \frac{d q}{q} \int_{0}^{1} d \nu\left(\frac{\sin ^{2}(\pi \nu)}{\pi^{2}}\right)\left(\frac{-\ln (q)}{2 \pi^{2}}\right)^{(k+1-D) / 2} \exp \left[16 l q^{2} \sin ^{4}(\pi \nu)\right]
$$

To extract the splitting rate from this amplitude we follow the work of [18] and change variables to $z=\sin ^{2}(\pi \nu)$ and $y=q^{2} z^{2}$. We are not interested in the singularities of the amplitude that arise when the two operators on the boundary coincide. So the entire analysis implicitly contains a cutoff that bounds $z$ away from zero and is removed after the rest of computations are carried out. Keeping this cutoff in mind so that $\ln \left(q^{2} z^{2}\right) \approx 2 \ln (q)$ for small $q$, the amplitude in the new variables becomes:

$$
A_{N}=\frac{\mathcal{N}_{N}}{2 \pi^{3}\left(2 \pi^{2}\right)} \int_{0}^{1} d z \frac{z}{\sqrt{z} \sqrt{1-z}} \int_{0}^{z^{2}} \frac{d y}{y}\left(\frac{-\ln (y)}{4 \pi^{2}}\right)^{\frac{k+1-D}{2}} e^{16 l y}
$$

The inner integral is only well-approximated by small values of $q$ if $l$ is large and negative. So we analytically continue the amplitude in the complex $l$ plane by setting $l \rightarrow-\bar{l}$. The $y$ integral can now be reliably estimated by approximating exp $-16 \bar{l} y$ as 1 for $0 \leq y \leq 1 /(16 \bar{l})$ and 0 for $y>1 /(16 \bar{l})$. The estimate of the analytically continued $y$ integral is:

$$
E=\frac{-2}{(k+3-D)}\left(\frac{1}{4 \pi^{2}}\right)^{\frac{k+1-D}{2}}(\ln 16 \bar{l})^{\frac{k+3-D}{2}}
$$

The integral over $z$ can now be done independently and gives $B(3 / 2,1 / 2)=\pi / 2$. Multiplying the factors together, rotating $l$ back to positive real line $(\bar{l} \rightarrow-\bar{l})$ and taking the leading imaginary part of the amplitude gives:

$$
\operatorname{Im}\left(A_{N}\right)=2 \pi^{2} \sqrt{l}\left(\frac{\ln (l)}{2 \pi}\right)^{\frac{k+1-D}{2}}
$$

where we used the normalization $\xi_{N}^{2}=1 / 2 \sqrt{l}$. The splitting rate is given by $\operatorname{Im}\left(A_{N}\right)$. We recover the result for a conventional open string by taking $k=9$. In that case, the decay rate is proportional to $\sqrt{l}$ as it should be for a splitting rate per unit length. For strings attached to a general k-brane the decay rate is suppressed by logarithms of the energy. These logarithms arose from the restriction to the Neumann directions 
of the momenta running around the loop. Equivalently, they arose from a truncation of intermediate open string states travelling normal to brane and the consequent restriction of the decay phase space.

An asymptotic analysis of this type would not have worked for the transversely polarized states. In that case the important region of the $\nu$ integral was in the neighbourhood of 0 and 1 which is precisely the region cut off in this analysis. Essentially, the dominant decays of states polarized parallel to the brane are into channels where both decay products are at low levels compared to the original state. These decays are allowed because angular momentum can be transferred to the relative spatial motion of the decay products and have a relatively large phase space. This is in marked contrast to the situation for transversely polarized states.

\section{Forces Between Branes}

The one-loop amplitude in Eq. 5 with nonzero $Y$ can be interpreted as the static interaction potential between a D-brane carrying massive excitations and a parallel Dbrane in its ground state a distance $Y^{\mu}$ away. This interpretation follows by thinking of the annulus diagram in the cross-channel as a closed string exchanged by D-branes in position eigenstates. To study long range forces we want to extract the contribution of the massless closed string states which arises from the $w \rightarrow 1$ limit of Eq. 5. This limit is most conveniently extracted as the $q \rightarrow 0$ limit in the annular variable $\ln q=2 \pi^{2} / \ln w$ introduced in Section 3.3.

Parallel Polarizations: The $q \rightarrow 0$ limit has already been extracted for parallel polarizations in Eq. 19, except that we must restore the factor of $\exp \left(Y^{2} / \ln q\right)$ that was dropped there. For small $q$ this term dominates over the exponential of $q^{2}$ that appeared in Eq. 19 so we can drop the latter term in computing long-range forces. Changing variables to $z=\sin ^{2}(\pi \nu)$ and $t=-1 / \ln (q)$ we find that the contribution of the closed string massless states is:

$$
A_{N}=\frac{\mathcal{N}_{N}}{2 \pi^{2} \pi^{3}} \pi^{3} \int_{0}^{1} \frac{d z z}{\sqrt{z} \sqrt{1-z}} \int_{0}^{\infty} \frac{d t}{t^{2}}\left(\frac{1}{2 \pi^{2} t}\right)^{\frac{k+1-D}{2}} e^{-t Y^{2}}
$$

Doing the integrals and putting in the normalizing factor $\mathcal{N}$ gives:

$$
A_{N}=2 \pi \sqrt{l}\left(\frac{1}{\pi}\right)^{\frac{k+1-D}{2}} \Gamma\left(\frac{D-3-k}{2}\right)\left(\frac{1}{Y^{2}}\right)^{\frac{D-3-k}{2}}
$$

$A_{N}$ is proportional to the mass of the excitation $\sqrt{l}$ times the $9-k$ dimensional Green's function $G_{9-k}\left(Y^{2}\right)$. Exciting the open string states on the first brane has broken the residual supersymmetry of the BPS state and this is reflected in a nonvanishing force. We see a long-range Coulombic force acting between the parallel branes that is proportional to $\sqrt{l}$, the mass excess over the BPS bound. The force vanishes for $l=0$ because parallel massless excitations of a brane do not destroy the BPS saturation. This provides a nice check on our result. 
Transverse Polarizations: Repeating the analysis of small $q$ asymptotics for transverse polarizations gives:[]

$A_{D}=\frac{\mathcal{N}_{D}}{2 \pi^{2}} \int_{0}^{1} d \nu \int_{0}^{1} \frac{d q}{q}\left(\frac{\sin ^{2}(\pi \nu)}{\pi^{2}}\right)\left(\frac{-\ln (q)}{2 \pi^{2}}\right)^{(k+1-D) / 2} \exp \left[\left(Y^{2}+2 l \sin ^{2}(\pi \nu)\right) / \ln (q)\right]$

Changing variables to $z$ and $t$ as above gives:

$$
A_{D}=\frac{\mathcal{N}_{D}}{2 \pi^{2} \pi^{3}} \int_{0}^{1} \frac{d z z}{\sqrt{z} \sqrt{1-z}} \int_{0}^{\infty} \frac{d t}{t^{2}}\left(\frac{1}{2 \pi^{2} t}\right)^{\frac{k+1-D}{2}} e^{-t\left(Y^{2}+2 l z\right)}
$$

Doing the integrals and inserting $\mathcal{N}_{D}$ gives a static interaction potential of:

$$
\begin{aligned}
A_{D} & =4 \sqrt{l}\left(\frac{1}{\pi}\right)^{\frac{k+1-D}{2}} \Gamma\left(\frac{D-3-k}{2}\right) \int_{0}^{1} \frac{d z z}{\sqrt{z} \sqrt{1-z}}\left(\frac{1}{Y^{2}+2 l z}\right)^{\frac{D-3-k}{2}} \\
& \propto \sqrt{l} \int_{0}^{1} \frac{d z z}{\sqrt{z} \sqrt{1-z}} G_{9-k}\left(Y^{2}+2 l z\right)
\end{aligned}
$$

As in the Neumann case the potential is proportional to the mass of the added open string state and to a $9-k$ dimensional Green's function. However, putting back the powers of $\alpha^{\prime}$, the argument of the Green's function is now $Y^{2} / 2 \alpha^{\prime}+2 l z$, and there remains an integral over $z$ running from 0 to 1 . In other words, the brane acts like an extended object with thickness of order $\sqrt{4 l \alpha^{\prime}}$ because of the remaining integral over $z$. It can be easily checked that this effect is not an artifact of the leading order in $q$ approximation. The static potential computed here does not depend on the relative orientation of the excitation and the separation $Y$ of the branes, since we are only considering states polarized orthogonal to $Y$. (See Section 2.) Indeed, the form of the potential is what is expected for a body with extent of order $\sqrt{4 l \alpha^{\prime}}$ transverse to the line of separation. This is a very direct demonstration of how the transversely polarized massive states endow D-branes with thickness. Note that our study of the longitudinally polarized states did not reveal this effect because such states lie entirely within the world volume.

\section{Conclusion and Discussion}

In this paper we have computed the one-loop diagram for leading Regge trajectory open string states attached to a D-brane. Generically, such states are expected to be quite unstable because they are far from BPS saturation. Nevertheless, we showed that there are large kinematic suppressions which can partially stabilize such systems. States that are polarized parallel to a brane have decay rates suppressed by powers of

\footnotetext{
${ }^{9}$ To arrive at this expression, we use standard $\theta$ function identities to find that in the small $q$ limit the leading term in the integrand of Eq. 0 contains a factor $\left(1+2 \sin ^{2}(\pi \nu) / \ln q\right)^{l}$. We write this as $\exp \left[l \ln \left(1+2 \sin ^{2}(\pi \nu) / \ln q\right)\right]$ and keep the leading term in the exponent. This treatment is valid for large $l$.
} 
logarithms of the energy. Remarkably, states polarized normal to a brane have leading decay rates that often decrease with energy. In particular, leading Regge trajectory states on 0-branes cannot decay by splitting into two lighter states and must rely on higher order processes to de-excite.

The splitting of strings discussed in this paper leads to equipartition of energy amongst the degrees of freedom living on the brane. In view of the large kinematic suppressions found above, it would be interesting to compute the rate for emission of closed strings in order to see whether a highly excited RR soliton has a chance to thermalize before a sizable portion of its energy is radiated away. It has been found that closed string emission from D-branes with massless excitations is exponentially suppressed in the energy of the emitted state [27]. This suggests that the power law suppression of equipartition found in this paper is not sufficient to impede thermalization. It would be very interesting to carry out similar analyses of the regular black hole configurations in [2] - [10].

The large suppressions found in this paper arise from restriction of momenta to lie parallel the brane and mean that the world volume decays of transversely polarized states may largely be ignored for very heavy states. The dominant decay mode for these states will be into a single outgoing massless closed string. This process may be computed on a disk with one boundary and one bulk operator or by looking at the closed string poles of the non-planar loop diagram. Sub-leading Regge trajectory decays will suffer less severe suppression. This is because, as discussed in Section 3.1, some of the one-loop kinematic constraints translate into angular momentum conservation in tree level diagrams. These constraints are less severe for sub-leading trajectories.

In Section 4 we have computed the static force between a brane excited by a leading Regge trajectory state and another in its ground state. The force is nonvanishing because the system is no longer BPS saturated. When the excited string is polarized normal to the brane the force law shows evidence that the brane acts like an extended body with size of order $\sqrt{\alpha^{\prime}}$. These excitations can also be expected to affect the tachyonic instability that occurs when a brane and anti-brane approach each other [28, 29]. It would be interesting to understand how exciting a brane affects this singularity.

\section{Acknowledgments}

We would like to thank Aki Hashimoto for collaboration in the early part of this project and Finn Larsen for fruitful discussions. We are also grateful to Curt Callan, Eric Sharpe and Larus Thorlacius for helpful conversations. V.B. was supported in part by DOE grant DE-FG02-91ER40671. I.R.K. was supported in part by DOE grant DE-FG02-91ER40671, the NSF Presidential Young Investigator Award PHY9157482, and the James S. McDonnell Foundation grant No. 91-48.

\section{References}


[1] J. Polchinski. Dirichlet-branes and Ramond-Ramond charges. Phys. Rev. Lett., 75:4724-4727, 1995.

[2] A. Strominger and C. Vafa. Microscopic origin of the Bekenstein-Hawking entropy. hep-th/9601029, January 1996.

[3] G.T. Horowitz and A. Strominger. Counting states of near-extremal black holes. hep-th/9602051, February 1996.

[4] A. Strominger. Macroscopic entropy of $\mathrm{N}=2$ extremal black holes. hepth/9602111, February 1996.

[5] J. Maldacena and A. Strominger. Statistical entropy of four-dimensional extremal black holes. hep-th/9603060, March 1996.

[6] J.C. Breckenridge, R.C. Myers, A.W. Peet, and C. Vafa. D-branes and spining black holes. hep-th/9602065, February 1996.

[7] C. Callan and J. Maldacena. D-brane approach to black hole quantum mechanics. hep-th/9602043, February 1996.

[8] C.V. Johnson, R.R. Khuri, and R.C. Myers. Entropy of 4D extremal black holes. hep-th/9603061, March 1996.

[9] I.R. Klebanov and A.A. Tseytlin. Intersecting M-branes as Four-Dimensional Black Holes. hep-th/9604160, April 1996.

[10] V. Balasubramanian and F. Larsen. On D-branes and black holes in four dimensions. hep-th/9604189, PUPT-1617, April 1996.

[11] G.T. Horowitz and A. Strominger. Black strings and p-branes. Nuc. Phys. B, 360:197-209, 1991.

[12] S.S. Gubser, I.R. Klebanov and A.W. Peet. Entropy and Temperature of Black 3-Branes. hep-th/9602135, February 1996.

[13] I.R. Klebanov and A. Tseytlin. Entropy of near-extremal black p-branes. hepth/9604089, April 1996.

[14] I.R. Klebanov and L. Thorlacius. The Size of p-Branes. hep-th/9610200, Phys. Lett. B, 371:51-56, 1996.

[15] S.S. Gubser, A. Hashimoto, I.R. Klebanov and J. Maldacena. Gravitational Lensing by p-branes. hep-th/960105 , January 1996.

[16] M.R. Garousi and R.C. Myers. Superstring Scattering from D-branes. hepth/9603194, March 1996.

[17] D. Mitchell, N. Turok, R. Wilkinson, and P. Jetzer. The decay of highly excited open strings. Nuc. Phys. B, 315:1-24, 1989. 
[18] D. Mitchell, B. Sundborg, and N. Turok. Decay of massive open strings. Nuc. Phys. B, 335:621-634, 1990.

[19] V.A. Kostelecky, O. Lechtenfeld, and S. Samuel. Covariant string amplitudes on exotic topologies to one loop. Nuc. Phys. B, 298:133-177, 1988.

[20] H. Yamamoto. One-loop mass shifts in $\mathrm{O}(32)$ open superstring theory. Prog. of Theoretical Physics, 79(1):189-208, January 1988.

[21] M.B. Green, J.H. Schwarz, and E. Witten. Superstring Theory. Cambridge University Press, 1987.

[22] J. Polchinski, S. Chaudhuri, and C.V. Johnson. Notes on D-Branes. hepth/9602059, February 1996.

[23] M. Gutperle. Multi-boundary effects in Dirichlet string theory. hep-th/9502100, February 1995.

[24] A. Cohen, G. Moore, P. Nelson, and J. Polchinski. Semi-off-shell string amplitudes. Nuc. Phys. B, 281:127-144, 1987.

[25] H. Okada and A. Tsuchiya. The decay rate of massive modes in type-I superstring. Phys. Lett. B, 232(1):91-95, 1989.

[26] E.T. Whittaker and G.N. Watson. A Course of Modern Analysis. Cambridge University Press, 1969.

[27] A. Hashimoto and I. Klebanov. Decay of excited D-branes. hep-th/9604065, April 1994.

[28] T. Banks and L. Susskind. Brane-Antibrane forces. hep-th/9511194, November 1995.

[29] M.B. Green and M. Gutperle. Light-cone supersymmetry and D-branes. hepth/9604091, April 1994. 